\title{
Low order controller design for systems with time delays
}

\author{
A. N. Gündeş and H. Özbay
}

\begin{abstract}
Finite-dimensional controller synthesis methods are developed for some classes of linear, time-invariant, singleinput single-output, or multi-input multi-output systems, which are subject to time delays. The proposed synthesis procedures give low-order stabilizing controllers that also achieve integralaction so that constant reference inputs are tracked asymptotically with zero steady-state error.
\end{abstract}

\section{INTRODUCTION}

A wide range of dynamical phenomena cannot be modeled sufficiently accurately as finite-dimensional linear timeinvariant (LTI) systems due to time delays. The effects of these delays often cannot be ignored and have to be included in the model [4], [7]. This paper presents finite-dimensional stabilizing controller synthesis methods for some classes of LTI, single-input single-output (SISO) or multi-input multioutput (MIMO) systems that are subject to time delays. The proposed controllers are simple and have low-order, and they also provide integral-action so that step-input references are tracked asymptotically with zero steady-state error.

The plant classes considered in Section III-A and Section III-B have no restrictions on their poles. These plants may be stable or unstable. The (transmission) zeros in the open left-half complex plane (OLHP) are unrestricted and there may be any number of zeros at infinity as well. The dual case in Section III-C considers plants with no restrictions on the number or location of the (transmission) zeros, but the poles are either in the OLHP or at the origin $s=0$. Section III-A examines SISO delayed plants of retarded type (e.g., [4], [1], [2]) and Theorem 1 develops a simple controller synthesis procedure, which is generalized and extended to MIMO systems in Theorem 2, Section III-B.

Stability of delay systems of retarded type and of neutral type was studied extensively and many delay-independent and delay-dependent stability results are available [7], [10]. The tuning and internal model control techniques used in process control systems generally apply to delay systems [12]. Infinite dimensional integral action controllers have been designed in [11] to maximize the allowable controller gain using the robust control techniques for infinite dimensional systems [5]. For MIMO stable plants subject to inputoutput delays, proportional-derivative (PD) and proportionalintegral-derivative (PID) controllers were designed in [8] for plants that have no more than two unstable poles close

Department of Electrical and Computer Engineering, University of California, Davis, CA 95616. angundes @ucdavis.edu

H. Özbay is with the Department of Electrical and Electronics Engineering, Bilkent University, Ankara, 06800 Turkey hitayabilkent.edu.tr to the origin. Arbitrary delay terms in addition to inputoutput delays were considered in [9] with decentralized controller structures. Restricting the designed controllers to be PD and PID imposes these restrictions on the number of unstable plant poles [13]. The results in this paper apply to much wider classes of SISO and MIMO systems by allowing the order of the controller to exceed that of PD or PID. The advantages of integral-action and simple low-order implementation are still part of the synthesis technique.

Notation: Let $\mathbb{C}, \mathbb{R}, \mathbb{R}_{+}$denote complex, real, and positive real numbers. The extended closed right-half complex plane is $\mathcal{U}=\{s \in \mathbb{C} \mid \mathcal{R} e(s) \geq 0\} \cup\{\infty\} ; \mathbf{R}_{\mathbf{p}}$ denotes real proper rational functions (of $s$ ); $\mathbf{S} \subset \mathbf{R}_{\mathbf{p}}$ is the stable subset with no poles in $\mathcal{U} ; \mathcal{M}(\mathbf{S})$ is the set of matrices with entries in $\mathbf{S} ; I_{r}$ is the $r \times r$ identity matrix. The space $\mathcal{H}_{\infty}$ is the set of all bounded analytic functions in $\mathbb{C}_{+}$. For $h \in \mathcal{H}_{\infty}$,

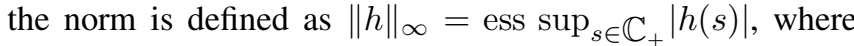
ess sup denotes the essential supremum. A matrix-valued function $H$ is in $\mathcal{M}\left(\mathcal{H}_{\infty}\right)$ if all its entries are in $\mathcal{H}_{\infty}$; in this case $\|H\|_{\infty}=\operatorname{ess} \sup _{s \in \mathbb{C}_{+}} \bar{\sigma}(H(s))$, where $\bar{\sigma}$ denotes the maximum singular value. Since all norms of interest here are $\mathcal{H}_{\infty}$ norms, we drop the norm subscript, i.e., $\|\cdot\|_{\infty} \equiv\|\cdot\|$. From the induced $L^{2}$ gain point of view, a system whose transfer-matrix is $H$ is stable iff $H \in \mathcal{M}\left(\mathcal{H}_{\infty}\right)$. A square transfer-matrix $H \in \mathcal{M}\left(\mathcal{H}_{\infty}\right)$ is unimodular iff $H^{-1} \in$ $\mathcal{M}\left(\mathcal{H}_{\infty}\right)$. We drop $(s)$ in transfer-matrices such as $G(s)$; use $\delta(n)$ to denote the degree of the polynomial $n(s)$; use $\operatorname{diag}\left[a_{\ell}\right]_{\ell=1}^{m}$ or $\operatorname{diag}\left[\begin{array}{llll}a_{1} & a_{2} & \cdots & a_{m}\end{array}\right]$ to denote the $(m \times m)$ diagonal matrix, whose diagonal entries are $a_{1}, \ldots, a_{m}$. For $G \in \mathbf{R}_{\mathbf{p}}{ }^{m \times m}$ we use coprime factorizations over $\mathbf{S}$; i.e., $G=Y^{-1} X$ denotes a left-coprime-factorization (LCF), where $X, Y \in \mathbf{S}^{m \times m}$, $\operatorname{det} Y(\infty) \neq 0$. For the delayed plant case, we use coprime factorizations over $\mathcal{H}_{\infty}$; i.e., $\widehat{G}=\widehat{Y}^{-1} \widehat{X}$ denotes a left-coprime-factorization (LCF), where $\widehat{X}, \widehat{Y} \in \mathcal{H}_{\infty}{ }^{m \times m}$.

\section{PROBlem DESCRIPTION}

Consider the feedback system $\operatorname{Sys}(\widehat{G}, C)$ in Fig. $1 ; C \in$ $\mathbf{R}_{\mathbf{p}}{ }^{m \times m}$ is the transfer-function of the controller and $\widehat{G}$ is the transfer-function of the plant with time delays. It is assumed that the feedback system is well-posed and the delay-free part of the plant (i.e, the plant without the time delay terms) and the controller have no unstable hiddenmodes. With $u, v, w, y$ as the input and output vectors, the closed-loop transfer-matrix $\widehat{H}$ from $(u, v)$ to $(w, y)$ is

$$
\widehat{H}=\left[\begin{array}{cc}
C(I+\widehat{G} C)^{-1} & -C(I+\widehat{G} C)^{-1} \widehat{G} \\
\widehat{G} C(I+\widehat{G} C)^{-1} & (I+\widehat{G} C)^{-1} \widehat{G}
\end{array}\right] .
$$

Let the (input-error) transfer-function from $u$ to $e$ be denoted 


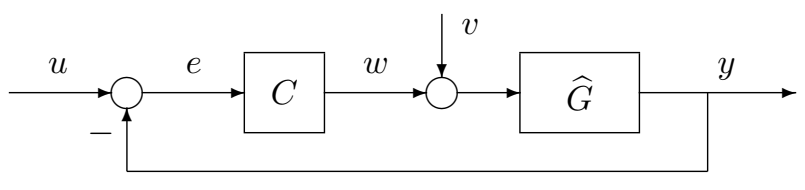

Fig. 1. The feedback system $\operatorname{Sys}(\widehat{G}, C)$.

by $H_{e u}$ and let the (input-output) transfer-function from $u$ to $y$ be denoted by $H_{y u}$; then

$$
H_{e u}=(I+\widehat{G} C)^{-1}=I-\widehat{G} C(I+\widehat{G} C)^{-1}=I-H_{y u} .
$$

Definition 1: a) The feedback system $\operatorname{Sys}(\widehat{G}, C)$ shown in Fig. 1, is stable if the closed-loop map $\widehat{H}$ is in $\mathcal{M}\left(\mathcal{H}_{\infty}\right)$. b) The controller $C$ stabilizes $\widehat{G}$ if $C$ is proper and $\operatorname{Sys}(\widehat{G}, C)$ is stable. c) The system $\operatorname{Sys}(\widehat{G}, C)$ is stable and has integralaction if the closed-loop transfer-function from $(u, v)$ to $(w, y)$ is stable, and the (input-error) transfer-function $H_{e u}$ has blocking-zeros at $s=0$. d) The controller $C$ is said to be an integral-action controller if $C$ stabilizes $\widehat{G}$ and $D(0)=0$ for any RCF $C=N D^{-1}$.

Let $\widehat{G}=\widehat{Y}^{-1} \widehat{X}$, where $\widehat{Y}, \widehat{X} \in \mathcal{M}\left(\mathcal{H}_{\infty}\right)$. Let $C=N D^{-1}$ be an RCF, where $D, N \in \mathbf{S}^{m \times m}$, $\operatorname{det} D(\infty) \neq 0$. Then $C$ stabilizes $\widehat{G}$ if and only if $M^{-1} \in \mathcal{M}\left(\mathcal{H}_{\infty}\right)$, where

$$
M:=\widehat{Y} D+\widehat{X} N .
$$

Suppose that the system $\operatorname{Sys}(\widehat{G}, C)$ is stable and that step input references are applied at $u(t)$. The steady-state error $e(t)$ due to step inputs at $u(t)$ goes to zero as $t \rightarrow \infty$ if and only if $H_{e u}(0)=0$. Therefore, by Definition 1-(c), the stable system $\operatorname{Sys}(\widehat{G}, C)$ achieves asymptotic tracking of constant reference inputs with zero steady-state error if and only if it has integral-action. By (3), write $H_{e u}=(I+\widehat{G} C)^{-1}=$ $D M^{-1} \widehat{Y}$. Then by Definition 1-(d), Sys $(\widehat{G}, C)$ has integralaction if $C=N D^{-1}$ is an integral-action controller since $D(0)=0$ implies $H_{e u}(0)=\left(D M^{-1} \widehat{Y}\right)(0)=0$. The system $\operatorname{Sys}(\widehat{G}, C)$ would also have integral-action if every entry of the MIMO plant has poles at $s=0$ since $\widehat{Y}(0)=0$ implies $H_{\text {eu }}(0)=0$ even if the controller's $D(0) \neq 0$. Therefore, it is not a necessary condition to have integral-action controllers for the system to have integral-action when $\widehat{Y}(0)=0$. However, for robust designs, integral-action is achieved with poles duplicating the dynamic structure of the exogenous signals that the regulator has to process; these integral-action controllers obey the well-known internal model principle [6].

We assume throughout that $\widehat{G}$ has no transmission-zeros at $s=0$. This condition is a necessary condition for existence of integral-action controllers: Let the $(m \times m)$ matrix $\widehat{G}(s)$ have (normal) $\operatorname{rank} G(s)=m$. If $\widehat{G}$ admits an integral-action controller, then it has no transmission-zeros at $s=0$.

\section{Controller SYNTHESis}

We propose finite-dimensional stabilizing controller synthesis for certain classes of plants that have time delays. The discussion in Section III-A applies to a class of SISO delay systems. In Section III-B, the results are extended to a class of MIMO plants with poles anywhere in the complex plane, but zeros restricted to be in the OLHP. Section III-C includes MIMO delay systems whose zeros are unrestricted, but the poles are either at the origin or in the stable region.

\section{A. SISO plants of retarded type}

We consider SISO delay plants described as

$$
\widehat{G}(s)=\frac{x(s)}{y(s)+q(s)}, \quad q(s)=\sum_{i=1}^{\nu} e^{-h_{i} s} q_{i}(s),
$$

where $x(s), y(s), q_{i}(s)$ are polynomials with real coefficients, $\delta(x) \leq \delta(y)>\delta\left(q_{i}\right)$, the integers $h_{i}>0, i=$ $1, \ldots, \nu$. We assume that the finite zeros of $\widehat{G}$ are in the OLHP, i.e, the polynomial $x(s)$ is strictly Hurwitz. Let $r:=\delta(y)-\delta(x) \geq 0$. Let $\xi(s)$ be any monic $r$-th order strictly Hurwitz polynomial; for example, $\xi=(s+a)^{r}$ for any $a \in \mathbb{R}_{+}$. Define

$$
\begin{aligned}
& Y_{n}:=\frac{y(s)}{x(s) \xi(s)}, \quad Y_{d}:=\frac{q(s)}{x(s) \xi(s)}, \\
& \widehat{Y}=Y_{n}+Y_{d}, \quad X:=\xi(s)^{-1} .
\end{aligned}
$$

Then $X, Y_{n} \in \mathbf{S}, Y_{d} \in \mathcal{H}_{\infty}$, and $\widehat{G}=(\widehat{Y})^{-1} X=\left(Y_{n}+\right.$ $\left.Y_{d}\right)^{-1} X$. Theorem 1 presents a finite-dimensional controller synthesis for closed-loop stability. This design gives integralaction controllers of order $r$ when the relative degree of $x(s) / y(s)$ is $r \geq 1$, or of order 1 when the relative degree of $x(s) / y(s)$ is zero.

Theorem 1: (SISO stabilizing controller synthesis): Let $\widehat{G}(s)$ be as in (4) For any monic $r$-th order strictly Hurwitz polynomial $\xi(s)$, let $\widehat{G}(s)=\widehat{Y}^{-1} X=\left(Y_{n}+Y_{d}\right)^{-1} X$ be as in (5). a) If $r=0$, then choose any $g \in \mathbb{R}_{+}$. Let $\alpha_{o} \in \mathbb{R}_{+}$ be such that

$$
\alpha_{o}>\left\|\frac{s}{s+g} \widehat{G}^{-1}\right\|=\left\|\frac{s}{s+g} \widehat{Y}\right\| .
$$

Then the controller $C_{o}$ in (7) stabilizes $\widehat{G}$ :

$$
C_{o}=\alpha_{o} \frac{(s+g)}{s} \text {. }
$$

b) If $r \geq 1$, then choose any monic, strictly Hurwitz polynomial $\xi(s)$ of order $r$. Define $\Theta$ as

$$
\begin{aligned}
\Theta(s):=s\left[\frac{1}{\xi(s)} \widehat{G}(s)^{-1} Y_{n}(\infty)^{-1}-1\right] & \\
\quad & s\left[\left(Y_{n}(s)+Y_{d}(s)\right) Y_{n}(\infty)^{-1}-1\right] .
\end{aligned}
$$

Let $\alpha \in \mathbb{R}_{+}$be such that

$$
\alpha>r\|\Theta(s)\| .
$$

Then the controller $C$ in (10) stabilizes $\widehat{G}$ :

$$
C=\frac{\alpha^{r} \xi(s)}{(s+\alpha)^{r}-\alpha^{r}} Y_{n}(\infty) .
$$

Remark: In Theorem 1, the SISO controllers $C_{o}$ in (7) for $r=0, C$ in (10) for $r \geq 1$ are biproper, and each has a pole at $s=0$ providing integral-action. The remaining $(r-1)$ poles of $r$-th order controller $C$ in (10) are all in the OLHP. Proof of Theorem 1: a) If $r=0$, then $X=1$ in $\widehat{G}=$ $\widehat{Y}^{-1} X$. Let $N=1, D=C_{o}^{-1}$; then $C_{o}=N D^{-1}$ is a 
coprime factorization of the proposed controller in (7). By (3), $C_{o}$ stabilizes $\widehat{G}$ if and only if $M^{-1} \in \mathcal{H}_{\infty}$, where

$$
M=X N+\widehat{Y} D=1+\widehat{Y}(s) \frac{s}{\alpha_{o}(s+g)} .
$$

Since $\alpha_{o}$ satisfies (6), $\left\|\widehat{Y}(s) \frac{s}{\alpha_{o}(s+g)}\right\|<1$, which is a sufficient condition for $M^{-1} \in \mathcal{H}_{\infty}$. Therefore, $C_{o}$ stabilizes $\widehat{G}$ since $M^{-1} \in \mathcal{H}_{\infty}$. b) Define $\varphi:=\left[(s+\alpha)^{r}-\alpha^{r}\right]$. Let $N=\alpha^{r}, D=\frac{\varphi(s)}{\xi(s)} Y_{n}(\infty)^{-1}$. Then $C=N D^{-1}$ is a coprime factorization of the proposed controller in (10). By (3), $C$ stabilizes $\widehat{G}$ if and only if $M^{-1} \in \mathcal{M}\left(\mathcal{H}_{\infty}\right)$, where

$$
\begin{aligned}
M & =X N+\widehat{Y} D=\frac{\alpha^{r}}{\xi(s)}+\widehat{Y}(s) \frac{\varphi(s)}{\xi(s)} Y_{n}(\infty)^{-1} \\
= & \left(\frac{\alpha^{r}}{(s+\alpha)^{r}}+\frac{\varphi(s)}{(s+\alpha)^{r}} \widehat{Y}(s) Y_{n}(\infty)^{-1}\right) \frac{(s+\alpha)^{r}}{\xi(s)} \\
= & \left(1+\frac{\varphi}{(s+\alpha)^{r}}\left[\widehat{Y}(s) Y_{n}(\infty)^{-1}-1\right]\right) \frac{(s+\alpha)^{r}}{\xi(s)} \\
& =\left(1+\frac{\varphi}{s(s+\alpha)^{r}} \Theta(s)\right) \frac{(s+\alpha)^{r}}{\xi(s)} .
\end{aligned}
$$

A sufficient condition for $M^{-1}$ to be in $\mathcal{M}\left(\mathcal{H}_{\infty}\right)$ is that $\left\|\frac{\varphi}{s(s+\alpha)^{r}} \Theta(s)\right\|<1$. We first show that

$$
\left\|\frac{\varphi}{s(s+\alpha)^{r}}\right\|=\left\|\frac{\left[(s+\alpha)^{r}-\alpha^{r}\right]}{s(s+\alpha)^{r}}\right\|=\frac{r}{\alpha} .
$$

For $r \geq 1,\left.\left(\frac{\left[(s+\alpha)^{r}-\alpha^{r}\right]}{s(s+\alpha)^{r}}\right)\right|_{s=0}=\frac{r}{\alpha}$ implies that the norm in (11) is greater than or equal to $r / \alpha$. We prove the norm in (11) is less than or equal to $r / \alpha$ by iteration: For $r=1,(11)$ holds since $\left\|\frac{s}{s(s+\alpha)}\right\|=1 / \alpha$. For $r=2,\left\|\frac{\left[(s+\alpha)^{2}-\alpha^{2}\right]}{s(s+\alpha)^{2}}\right\|=$ $\left\|\frac{\left[s^{2}+2 \alpha s\right]}{s(s+\alpha)^{2}}\right\|=2 / \alpha$. For $r=3,\left\|\frac{1}{s+\alpha}\right\|=1 / \alpha$ implies

$$
\begin{gathered}
\left\|\frac{\left[(s+\alpha)^{3}-\alpha^{3}\right]}{s(s+\alpha)^{3}}\right\|=\| \frac{1}{(s+\alpha)} \frac{\left[(s+\alpha)(s+\alpha)^{2}-\alpha \alpha^{2}\right]}{s(s+\alpha)^{2}} \\
\leq\left\|\frac{1}{s+\alpha}\right\|\left\|\frac{\alpha\left[(s+\alpha)^{2}-\alpha^{2}\right]}{s(s+\alpha)^{2}}+1\right\| \\
\leq \frac{1}{\alpha}\left[\left\|\frac{\alpha\left[(s+\alpha)^{2}-\alpha^{2}\right]}{s(s+\alpha)^{2}}\right\|+1\right]=\frac{1}{\alpha}\left[\alpha \frac{2}{\alpha}+1\right]=\frac{3}{\alpha},
\end{gathered}
$$

hence, (11) holds. Continuing similarly, suppose that (11) holds for $r$ and show that it holds for $(r+1)$ :

$$
\begin{aligned}
\left\|\frac{\left[(s+\alpha)^{r+1}-\alpha^{r+1}\right]}{s(s+\alpha)^{r+1}}\right\|=\left\|\frac{\left[(s+\alpha)(s+\alpha)^{r}-\alpha \alpha^{r}\right]}{(s+\alpha) s(s+\alpha)^{r}}\right\| \\
\leq\left\|\frac{1}{s+\alpha}\right\|\left\|\frac{\alpha \varphi}{s(s+\alpha)^{r}}+1\right\| \leq \frac{1}{\alpha}\left[\left\|\frac{\alpha \varphi}{s(s+\alpha)^{r}}\right\|+1\right] \\
=\frac{1}{\alpha}\left[\alpha \frac{r}{\alpha}+1\right]=\frac{r+1}{\alpha},
\end{aligned}
$$

hence, (11) holds. In (8), $Y_{n}(s) Y_{n}(\infty)^{-1}=1$ implies $s\left[Y_{n}(s) Y_{n}(\infty)^{-1}-I\right] \in \mathbf{S}$. Since $\delta(q)<\delta(y)=\delta(x \xi)$, we have $s Y_{d}(s) Y_{n}(\infty)^{-1} \in \mathcal{H}_{\infty}$. Therefore, $\Theta(s)=$ $s\left[Y_{n}(s) Y_{n}(\infty)^{-1}-I\right]+s Y_{d}(s) Y_{n}(\infty)^{-1} \in \mathcal{H}_{\infty}$. Since $\alpha$ satisfies (9), $\left\|\frac{\varphi}{s(s+\alpha)^{r}} \Theta(s)\right\| \leq \frac{r}{\alpha}\|\Theta(s)\|<1$. Therefore, $C$ in (10) stabilizes $\widehat{G}$ since $M^{-1} \in \mathcal{H}_{\infty}$.

\section{B. MIMO plants with unrestricted poles}

We consider $(m \times m)$ MIMO plants with delay, where the delays are all in the denominator matrix $\widehat{Y} \in \mathcal{M}\left(\mathcal{H}_{\infty}\right)$ of $\widehat{G}=\widehat{Y}^{-1} \widehat{X}$. Therefore, $\widehat{X}$ is delay-free and we denote it by $X \in \mathcal{M}(\mathbf{S})$. We assume that $\widehat{G}$ can be written as

$$
\begin{gathered}
\widehat{G}=\widehat{Y}^{-1} X ; \widehat{Y}=Y_{n}+Y_{d}, Y_{n}(s) \in \mathcal{M}(\mathbf{S}), \\
\operatorname{det} Y_{n}(\infty) \neq 0, Y_{d}=\sum_{i=1}^{\nu} e^{-h_{i} s} Q_{i}(s), Q_{i}(\infty)=0 .
\end{gathered}
$$

We assume that the transmission-zeros of $\widehat{G}$ are all in the OLHP and at infinity, i.e., $\operatorname{rank} X(\infty)<m$ but $\operatorname{rank} X(s)=$ $m$ for all $s \in \mathbb{C}_{+}$. With $n_{k \ell}$ and $d_{k \ell}$ as polynomials, write

$$
X^{-1}(s)=\left[\frac{n_{k \ell}(s)}{d_{k \ell}(s)}\right]_{k, \ell \in\{1, \ldots, m\}} .
$$

Since the transmission-zeros of $\widehat{G}$ are all in the OLHP, $X^{-1}$ has no poles in the closed right-half complex plane $\mathbb{C}_{+}$(i.e., $d_{k \ell}$ are strictly Hurwitz) but may have poles at infinity, i.e., $X^{-1}$ may be improper. Define the integers $r_{k \ell}$ and $r_{\ell}$ as

$$
\begin{aligned}
& r_{k \ell}:= \begin{cases}\delta\left(n_{k \ell}\right)-\delta\left(d_{k \ell}\right), & \text { if } \delta\left(n_{k \ell}\right)>\delta\left(d_{k \ell}\right) \\
0, & \text { if } \delta\left(n_{k \ell}\right) \leq \delta\left(d_{k \ell}\right)\end{cases} \\
& r_{\ell}:=\max _{1 \leq k \leq m} r_{k \ell}, \quad \ell=1, \ldots, m .
\end{aligned}
$$

Let $\xi_{\ell}(s)$ be any monic $r_{\ell}$-th order strictly Hurwitz polynomial, $\ell=1, \ldots, m$; e.g., $\xi_{\ell}(s)=(s+a)^{r_{\ell}}$ for $a \in \mathbb{R}_{+}$. Define

$$
\Delta(s):=\operatorname{diag}\left[\xi_{1}(s) \quad \xi_{2}(s) \quad \cdots \quad \xi_{m}(s)\right] .
$$

If $r_{\ell}=0$, then $\xi_{\ell}=1$. Although $X^{-1}$ may be improper, $X^{-1} \Delta^{-1}$ is stable since $\frac{n_{k \ell}(s)}{d_{k \ell}(s) \xi_{\ell}(s)} \in \mathbf{S}$. Define $\widehat{Y}(\infty):=\left.\left(X(s) \widehat{G}(s)^{-1}\right)\right|_{s \rightarrow \infty}$; i.e., $Y_{j}(\infty)^{-1}=$ $\left.(\widehat{G}(s) X-1(s))\right|_{s \rightarrow \infty}$. By (12), $\widehat{Y}(\infty)=Y_{n}(\infty)$.

For this class of MIMO plants, Theorem 2 presents a finite-dimensional controller synthesis with integral-action.

Theorem 2: (MIMO stabilizing controller synthesis): Let $\widehat{G}=\widehat{Y}^{-1} X=\left(Y_{n}+Y_{d}\right)^{-1} X$ be as in (12). Define $\Theta$ as

$$
\Theta(s):=s\left[\widehat{Y}(s) Y_{n}(\infty)^{-1}-I\right] .
$$

For $\ell=1, \ldots, m$, define $\rho_{\ell}$ as

$$
\rho_{\ell}:= \begin{cases}1, & \text { if } r_{\ell}=0 \\ r_{\ell}, & \text { if } r_{\ell} \geq 1\end{cases}
$$

Let $\alpha \in \mathbb{R}_{+}$be such that

$$
\alpha>\max _{\ell} \rho_{\ell}\|\Theta\| \text {. }
$$

For $\ell=1, \ldots, m$, define $\varphi_{\ell}$ as

$$
\varphi_{\ell}(s):=\left[(s+\alpha)^{\rho_{\ell}}-\alpha^{\rho_{\ell}}\right] .
$$

Then the controller $C$ in (20) stabilizes $\widehat{G}$ :

$C=X^{-1}(s) \operatorname{diag}\left[\begin{array}{llll}\frac{\alpha^{\rho_{1}}}{\varphi_{1}(s)} & \frac{\alpha^{\rho_{2}}}{\varphi_{2}(s)} & \cdots & \frac{\alpha^{\rho_{m}}}{\varphi_{m}(s)}\end{array}\right] Y_{n}(\infty)$. 
Remark: In Theorem 2, the diagonal terms of diag $\left[\frac{\alpha^{\rho_{\ell}}}{\varphi_{\ell}}\right]_{\ell=1}^{m}$ in the controller $C$ in (20) all have poles at $s=0$ and hence, $C$ has integral-action. The terms corresponding to $r_{\ell}=0$ are in the form $\frac{\alpha}{s}$. The terms corresponding to $r_{\ell} \geq 1$ are in the form $\left(\frac{\alpha^{r} \ell}{(s+\alpha)^{r} \ell-\alpha^{r} \ell}\right)$, with one pole at $s=0$, and the remaining $\left(r_{\ell}-1\right)$ poles all in the OLHP.

Proof of Theorem 2: For $\ell=1, \ldots, m$, define $\eta_{\ell}$ as

$$
\eta_{\ell}:=\frac{1}{(s+\alpha)^{\rho_{\ell}-r_{\ell}}} ;
$$

$\eta_{\ell}=\frac{1}{(s+\alpha)}$ if $r_{\ell}=0$, and $\eta_{\ell}=1$ if $r_{\ell} \geq 1$. If $r_{\ell}=0$, then let $\xi_{\ell}=1$. If $r_{\ell} \geq 1$, then choose any monic, strictly Hurwitz polynomial $\xi_{\ell}(s)$ of order $r_{\ell}$. With $\Delta$ as in (15), let

$$
\begin{aligned}
& N=X^{-1} \Delta^{-1} \operatorname{diag}\left[\begin{array}{llll}
\eta_{1} \alpha^{\rho_{1}} & \eta_{2} \alpha^{\rho_{2}} & \cdots & \eta_{m} \alpha^{\rho_{m}}
\end{array}\right] \\
& =X^{-1} \operatorname{diag}\left[\frac{\alpha^{\rho_{1}}}{\xi_{\ell}(s)(s+\alpha)^{\rho_{\ell}-r_{\ell}}}\right]_{\ell=1}^{m}, \\
& D=Y_{n}(\infty)^{-1} \Delta^{-1} \operatorname{diag}\left[\eta_{\ell} \varphi_{\ell}(s)\right]_{\ell=1}^{m} \\
& =Y_{n}(\infty)^{-1} \operatorname{diag}\left[\frac{\varphi_{\ell}(s)}{\xi_{\ell}(s)(s+\alpha)^{\rho_{\ell}-r_{\ell}}}\right]_{\ell=1}^{m} .
\end{aligned}
$$

Then $C=N D^{-1}$ is a right-factorization of the proposed controller in (20). By (3), $C$ stabilizes $\widehat{G}$ if and only if $M^{-1} \in \mathcal{M}\left(\mathcal{H}_{\infty}\right)$, where

$$
\begin{gathered}
M=X N+\widehat{Y} D=X X^{-1} \Delta^{-1} \operatorname{diag}\left[\alpha^{\rho_{\ell}} \eta_{\ell}\right]_{\ell=1}^{m} \\
+\widehat{Y}(s) Y_{n}(\infty)^{-1} \Delta^{-1} \operatorname{diag}\left[\eta_{\ell} \varphi_{\ell}\right]_{\ell=1}^{m} \\
=\operatorname{diag}\left[\frac{\alpha^{\rho_{\ell}}}{\xi_{\ell}(s)(s+\alpha)^{\rho_{\ell}-r_{\ell}}}\right]_{\ell=1}^{m} \\
+\widehat{Y}(s) Y_{n}(\infty)^{-1} \operatorname{diag}\left[\frac{\varphi_{\ell}(s)}{\xi_{\ell}(s)(s+\alpha)^{\rho_{\ell}-r_{\ell}}}\right]_{\ell=1}^{m} \\
\left.=\left(\operatorname{diag}\left[\frac{\alpha^{\rho_{\ell}}}{(s+\alpha)^{\rho_{\ell}}}\right]_{\ell=1}^{m}\right]^{m}\right) \operatorname{diag}\left[\frac{(s+\alpha)^{r_{\ell}}}{\xi_{\ell}(s)}\right]_{\ell=1}^{m} \\
\left.+\widehat{Y}(s) Y_{n}(\infty)^{-1} \operatorname{diag}\left[\frac{\varphi_{\ell}(s)}{(s+\alpha)^{r_{\ell}}}\right]_{\ell=1}^{m}\right]_{\ell=1}^{\varphi_{\ell}} \\
=\left(I+\left[\widehat{Y}(s) Y_{n}(\infty)^{-1}-I\right] \operatorname{diag}\left[\frac{(s+\alpha)^{\rho_{\ell}}}{(s+\alpha)^{r_{\ell}}}\right]_{\ell=1}^{m}\right]_{\ell=1}^{\xi_{\ell}(s)} .
\end{gathered}
$$

The entries of $\operatorname{diag}\left[\frac{\varphi_{\ell}(s)}{s(s+\alpha)^{\rho_{\ell}}}\right]_{\ell=1}^{m}$ for $r_{\ell}=0$ have norm $\left\|\frac{1}{(s+\alpha)}\right\|=\frac{1}{\alpha}$. The entries for $r_{\ell} \geq 1$ have norm

$$
\left\|\frac{\left[(s+\alpha)^{r_{\ell}}-\alpha_{\ell}^{r}\right]}{s(s+\alpha)^{r_{\ell}}}\right\|=\frac{r_{\ell}}{\alpha}=\frac{\rho_{\ell}}{\alpha}
$$

as shown in the proof of Theorem 1. Therefore,

$$
\left\|\operatorname{diag}\left[\frac{\varphi_{\ell}(s)}{s(s+\alpha)^{\rho_{\ell}}}\right]_{\ell=1}^{m}\right\|=\max _{\ell} \frac{\rho_{\ell}}{\alpha} .
$$

In $(16), s Y_{n}(s) Y_{n}(\infty)^{-1} \operatorname{diag}\left[\frac{\Delta_{\ell}}{\chi_{\ell}}\right]_{\ell=1}^{m} \in \mathcal{M}(\mathbf{S})$ since $Y_{n}(s) Y_{n}(\infty)^{-1}=I$. Since $Q_{i}(\infty)=0$ by (12), $s Y_{d}(s) Y_{n}(\infty)^{-1} \in \mathcal{M}\left(\mathcal{H}_{\infty}\right)$; hence, $\Theta \in \mathcal{H}_{\infty}$. Therefore $C$ stabilizes $\widehat{G}$ since $M^{-1} \in \mathcal{M}\left(\mathcal{H}_{\infty}\right)$ if

$$
\left\|\Theta(s) \operatorname{diag}\left[\frac{\varphi_{\ell}(s)}{s(s+\alpha)^{\rho_{\ell}}}\right]_{\ell=1}^{m}\right\| \leq\|\Theta(s)\| \max _{\ell} \frac{\rho_{\ell}}{\alpha}<1,
$$

which holds since $\alpha$ satisfies (18).

\section{MIMO plants with unrestricted transmission-zeros}

We consider $(m \times m)$ MIMO plants with delay, where the delays are all in the numerator matrix $\widehat{X} \in \mathcal{M}\left(\mathcal{H}_{\infty}\right)$ of $\widehat{G}=\widehat{Y}^{-1} \widehat{X}$, i.e., the denominator matrix $\widehat{Y}$ is delay-free and we denote it by $Y \in \mathcal{M}(\mathbf{S})$. Therefore, we assume that a left-coprime factorization of $\widehat{G}$ can be written as

$$
\widehat{G}=Y^{-1} \widehat{X} ; \widehat{X}_{i j}=e^{-h_{i j} s} X_{i j} ; i, j=1, \ldots, m ;
$$

the integers $h_{i j} \geq 0 ; Y \in \mathcal{M}(\mathbf{S})$ is delay-free; $\widehat{X} \in$ $\mathcal{M}\left(\mathcal{H}_{\infty}\right)$ and $\widehat{X}_{i j}$ denotes its $i j$-th entry. Suppose that each $i j$-th entry $\widehat{X}_{i j}$ of $\widehat{X}$ may contain any arbitrary delay terms and that the delays are known. If the finite-dimensional part $Y^{-1}$ of the delayed plant $\widehat{G}$ is stable, then (23) implies that the entries of $\widehat{G}$ may contain all different arbitrary known delay terms. Let $\widehat{G}$ have full (normal) rank $m$. Let $\widehat{G}$ have no transmission zeros at $s=0$, equivalently, $\operatorname{rank} \widehat{X}(0)=m$. We also assume that $Y^{-1}$ may have poles anywhere in the OLHP, but the only $\mathcal{U}$-poles of are all at $s=0$, i.e., the only $\mathbb{C}_{+}$-poles of $Y^{-1}$ are at the origin. The entries of $Y^{-1}$ may have different multiplicities of poles at $s=0$ and some entries may have only poles in the stable region $\mathbb{C} \backslash \mathcal{U}$. Write

$$
Y^{-1}(s)=\left[Y_{k \ell}(s)\right]_{k, \ell=1, \ldots, m} .
$$

For $\ell=1, \ldots, m$, define the integers $\gamma_{k \ell} \geq 0$ be the number of poles of $Y_{k \ell}(s)$ at $s=0$, and define $\gamma_{\ell}$ as

$$
\gamma_{\ell}:=\max _{1 \leq k \leq m} \gamma_{k \ell}
$$

i.e., $\gamma_{\ell} \geq 0$ is the largest number of poles at $s=0$ of the entries in the $\ell$-th column of $Y^{-1}(s)$. For $\ell=1, \ldots, m$, although $Y_{k \ell}(s) \notin \mathbf{S},\left(Y_{k \ell}(s) \frac{s^{\gamma} \ell}{(s+\beta)^{\gamma} \ell}\right) \in \mathbf{S}$ for any $\beta \in \mathbb{R}_{+}$.

For this class of (MIMO or SISO) plants, Theorem 3 presents a finite-dimensional controller synthesis; Corollary 1 includes integral-action in the stabilizing controller synthesis.

Theorem 3: (MIMO stabilizing controller synthesis): Let $\widehat{G}=Y^{-1} \widehat{X}$ be as in (23). Define $\Phi$ as

$$
\Phi(s):=\frac{1}{s}\left[\widehat{X}(s) X(0)^{-1}-I\right] .
$$

Choose $\beta \in \mathbb{R}_{+}$such that

$$
\beta<\frac{1}{\max _{\ell} \gamma_{\ell}}\|\Phi(s)\|^{-1} \text {. }
$$

For $\ell=1, \ldots, m$, define

$$
\psi_{\ell}(s):=\left[(s+\beta)^{\gamma_{\ell}}-s^{\gamma_{\ell}}\right] .
$$

Then the controller $C$ in (29) stabilizes $\widehat{G}$ :

$$
C=X(0)^{-1}\left[\begin{array}{cccc}
\frac{\psi_{1}(s)}{s^{\gamma}} & 0 & \ldots & 0 \\
0 & \frac{\psi_{2}(s)}{s^{\gamma_{2}}} & \ldots & 0 \\
& & \ddots & \\
0 & 0 & \ldots & \frac{\psi_{m}(s)}{s^{\gamma} m}
\end{array}\right] Y(s)
$$


Corollary 1: (Integral-action controller synthesis): Under the assumptions of Theorem 3 , choose $\beta \in \mathbb{R}_{+}$such that

$$
\beta<\frac{1}{1+\max _{\ell} \gamma_{\ell}}\|\Phi(s)\|^{-1} .
$$

For $\ell=1, \ldots, m$, define

$$
\tilde{\psi}_{\ell}(s):=\left[(s+\beta)^{1+\gamma_{\ell}}-s^{1+\gamma_{\ell}}\right] .
$$

Then the integral-action controller $\widetilde{C}$ in (32) stabilizes $\widehat{G}$ :

$$
\widetilde{C}=X(0)^{-1}\left[\begin{array}{cccc}
\frac{\tilde{\psi}_{1}(s)}{s^{1+\gamma_{1}}} & 0 & \ldots & 0 \\
0 & \frac{\tilde{\psi}_{2}(s)}{s^{1+\gamma_{2}}} & \ldots & 0 \\
& & \ddots & \\
0 & 0 & \ldots & \frac{\tilde{\psi}_{m}(s)}{s^{1+\gamma m}}
\end{array}\right] Y(s)
$$

Proof of Theorem 3: Let

$$
\Psi(s):=\operatorname{diag}\left[\begin{array}{llll}
\frac{\psi_{1}(s)}{(s+\beta)^{\gamma_{1}}} & \frac{\psi_{2}(s)}{(s+\beta)^{\gamma_{2}}} & \cdots & \frac{\psi_{m}(s)}{(s+\beta)^{\gamma m}}
\end{array}\right] .
$$

Define $N:=X(0)^{-1} \Psi(s)$,

$$
D:=Y^{-1} \operatorname{diag}\left[\begin{array}{llll}
\frac{s^{\gamma_{1}}}{(s+\beta)^{\gamma_{1}}} & \frac{s^{\gamma_{2}}}{(s+\beta)^{\gamma_{2}}} & \cdots & \frac{s^{\gamma_{m}}}{(s+\beta)^{\gamma_{m}}}
\end{array}\right] .
$$

Since the order of the polynomial $\psi_{\ell}(s)$ is $\left(\gamma_{\ell}-1\right)$, the strictly-proper terms $\frac{\psi_{\ell}(s)}{(s+\beta)^{\gamma}} \in \mathbf{S}$ are stable and hence, $N=X(0)^{-1} \Psi(s) \in \mathcal{M}(\mathbf{S})$. Since $\left(Y_{k \ell}(s) \frac{s^{\gamma_{\ell}}}{(s+\beta)^{\gamma_{\ell}}}\right) \in \mathbf{S}$, the matrix $D \in \mathcal{M}(\mathbf{S})$. Therefore, $C=N D^{-1}$ is a rightfactorization of the proposed controller in (29). By (3), $C$ stabilizes $\widehat{G}$ if and only if $M^{-1} \in \mathcal{M}\left(\mathcal{H}_{\infty}\right)$, where

$$
\begin{gathered}
M=\widehat{X} N+Y D=\widehat{X}(s) X(0)^{-1} \Psi(s) \\
+Y(s) Y^{-1}(s) \operatorname{diag}\left[\frac{s^{\gamma_{\ell}}}{(s+\beta)^{\gamma_{\ell}}}\right]_{\ell=1}^{m} \\
=\widehat{X}(s) X(0)^{-1} \Psi+\operatorname{diag}\left[\frac{s^{\gamma_{\ell}}}{(s+\beta)^{\gamma_{\ell}}}\right]_{\ell=1}^{m} \\
=\left[\widehat{X}(s) X(0)^{-1}-I\right] \Psi(s)=\frac{1}{s}\left[\widehat{X}(s) X(0)^{-1}-I\right] s \Psi(s) \\
=\Phi(s) s \Psi(s) .
\end{gathered}
$$

A sufficient condition for $M^{-1}$ to be in $\mathcal{M}\left(\mathcal{H}_{\infty}\right)$ is that $\|\Phi(s) s \Psi(s)\|<1$. To find $\|s \Psi(s)\|$, we first show that

$$
\left\|\frac{s \psi_{\ell}(s)}{(s+\beta)^{\gamma_{\ell}}}\right\|=\left\|\frac{s\left[(s+\beta)^{\gamma_{\ell}}-s^{\gamma_{\ell}}\right]}{(s+\beta)^{\gamma_{\ell}}}\right\|=\beta \gamma_{\ell} \text {. }
$$

For $\gamma_{\ell}=0$, (35) obviously holds. For $\gamma_{\ell} \geq 1$, $\left.\left(\frac{s\left[(s+\beta)^{\gamma_{\ell}}-s^{\gamma_{\ell}}\right]}{(s+\beta)^{\gamma_{\ell}}}\right)\right|_{s=\infty}=\beta \gamma_{\ell}$ implies that the norm in (35) is greater than or equal to $\beta \gamma_{\ell}$. We prove the norm in (35) is less than or equal to $\beta \gamma_{\ell}$. For $\gamma_{\ell}=1$, (35) holds since $\left\|\frac{\beta s}{s+\beta}\right\|=\beta$. For $\gamma_{\ell}=2,\left\|\frac{s\left[(s+\beta)^{2}-s^{2}\right]}{(s+\beta)^{2}}\right\|=\left\|\frac{s\left[2 \beta s+\beta^{2}\right]}{(s+\beta)^{2}}\right\|=$ $2 \beta$ and hence, (35) holds. For $\gamma_{\ell}=3,\left\|\frac{s}{s+\beta}\right\|=1$ implies $\left\|\frac{s\left[(s+\beta)^{3}-s^{3}\right]}{(s+\beta)^{3}}\right\| \leq\left\|\frac{s}{s+\beta}\right\|\left[\left\|\frac{s\left[(s+\beta)^{2}-s^{2}\right]}{(s+\beta)^{2}}\right\|+\beta\right]=(2 \beta+\beta)=$ $3 \beta$ and hence, (35) holds. Continuing similarly, suppose that (35) holds for $\gamma_{\ell}$ and show that it holds for $\left(\gamma_{\ell}+1\right)$ :

$$
\begin{aligned}
\left\|\frac{s\left[(s+\beta)^{\gamma_{\ell}+1}-s^{\gamma_{\ell}+1}\right]}{(s+\beta)^{\gamma_{\ell}+1}}\right\| \leq & \left\|\frac{s}{s+\beta}\right\|\left[\left\|\frac{s \psi_{\ell}(s)}{(s+\beta)^{\gamma_{\ell}}}\right\|+\beta\right] \\
& =\left(\beta \gamma_{\ell}+\beta\right)=\beta\left(\gamma_{\ell}+1\right)
\end{aligned}
$$

and hence, (35) holds. Now (35) implies $\|s \Psi(s)\|=$ $\beta \max _{\ell} \gamma_{\ell}$. Since $\beta$ satisfies (27), $\|\Phi(s) s \Psi(s)\| \leq$ $\|\Phi(s)\|\|s \Psi(s)\|=\beta \max \gamma_{\ell}\|\Phi(s)\|<1$. Therefore, $C$ stabilizes $\widehat{G}$ since $M^{-1} \in \mathcal{M}\left(\mathcal{H}_{\infty}\right)$.

Proof of Corollary 1: Let

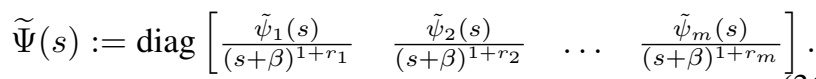

With $D$ as in (34), let $\widetilde{N}=X(0)^{-1} \widetilde{\Psi}(s), \widetilde{D}=\frac{s}{(s+\beta)} D$. Then $\widetilde{C}=\widetilde{N} \widetilde{D}^{-1}$ is a right-factorization of the proposed controller in (32); since $\widetilde{D}(0)=0$, by Definition $1-(\mathrm{d})$, $\widetilde{C}$ is an integral-action controller. We show that by (3), $\widetilde{C}$ stabilizes $\widehat{G}$ if and only if $\widetilde{M}^{-1} \in \mathcal{M}\left(\mathcal{H}_{\infty}\right)$, where $\widetilde{M}=$ $\widehat{X} \widetilde{N}+Y \widetilde{D}=\widehat{X} \widetilde{N}+\frac{s}{(s+\beta)} Y D=\widehat{X}(s) X(0)^{-1} \widetilde{\Psi}(s)+$ $\frac{s}{(s+\beta)} \operatorname{diag}\left[\frac{s^{r} \ell}{(s+\beta)^{r} \ell}\right]_{\ell=1}^{m}=\left[\widehat{X}(s) X(0)^{-1}-I\right] \widetilde{\Psi}(s)=$ $\frac{1}{s}\left[\widehat{X}(s) X(0)^{-1}-I\right] s \widetilde{\Psi}(s)=\Phi(s) s \widetilde{\Psi}(s)$. A sufficient condition for $\widetilde{M}^{-1} \in \mathcal{M}\left(\mathcal{H}_{\infty}\right)$ is $\|\Phi(s) s \widetilde{\Psi}(s)\|<1$, where, by (35), $\|s \widetilde{\Psi}(s)\|=\beta\left(1+\max \gamma_{\ell}\right)$ Since $\beta$ satisfies (30), $\|\Phi(s) s \widetilde{\Psi}(s)\| \leq\|\Phi(s)\|\|s \widetilde{\Psi}(s)\|=\beta(1+$ $\left.\max _{\ell} \gamma_{\ell}\right)\|\Phi(s)\|<1$. Therefore, the integral-action controller $\widetilde{C}^{\ell}$ stabilizes $\widehat{G}$ since $\widetilde{M}^{-1} \in \mathcal{M}\left(\mathcal{H}_{\infty}\right)$.

\section{EXAMPLES}

Example 1: Consider

$$
\begin{aligned}
& \widehat{G}(s)=\frac{(s+1)}{\left(s^{2}-2 s+2\right)+2(s-1) e^{-h_{1} s}+5 e^{-h_{2} s}} \\
&=\frac{x(s)}{y(s)+q(s)}, \quad h_{2}=\frac{\pi}{2} h_{1} .
\end{aligned}
$$

The plant $\widehat{G}$ is in the class considered in Section III-A. Since the relative degree $r=1$, the controller as in (10) is a first order controller with integral action (i.e. a PI controller). Let $\xi(s)=(s+b)$ for a free parameter $b>0$; define

$Y_{n}(s)=\frac{\left(s^{2}-2 s+2\right)}{(s+1)(s+b)}, Y_{d}(s)=\frac{2(s-1) e^{-h_{1} s}+5 e^{-h_{2} s}}{(s+1)(s+b)}$.

With $\Theta$ as in (8),

$$
\Theta(s)=\frac{s\left[\left(2(s-1) e^{-h_{1} s}+5 e^{-h_{2} s}\right)-(3+b) s-b+2\right]}{(s+1)(s+b)},
$$

the minimum value $\|\Theta(s)\|$ of $\alpha$ satisfying (9) is shown in Fig. 2 for various $h_{1}$. For $h_{1} \in[0.1,2.5]$, if we choose $b=$ 2 , then $\alpha=8$ satisfies (9). The controller in (10) is $C(s)=$ $\frac{8(s+2)}{s}$. This feedback system is stable if the transformed characteristic equation $1+\frac{1}{(s+\alpha)} \Theta(s)=0$ has no roots in the closed right half plane. Since $\|\Theta\|_{\infty}<\alpha$ and $\|(s+$ $\alpha)^{-1} \|_{\infty}=1 / \alpha$, the small gain theorem implies stability. Now change the plant $\widehat{G}$ in (37) to

$$
\widehat{G}_{w}=\frac{x(s)}{(s-p)^{w} y(s)+q(s)},
$$

$w>0, p \in \mathbb{R}$, and $x(s), y(s), q(s)$ are the same as in (37). The relative degree becomes $r=w+1$, and the delayed part 


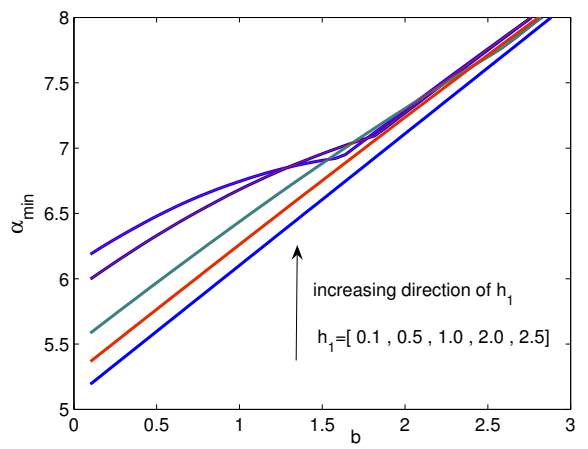

Fig. 2. Example 1: $b$ versus $\|\Theta\|$ for various $h_{1}$.

$Y_{d}(s)$ of $\widehat{Y}(s)$ remains the same. The new $Y_{n}$ is

$$
Y_{n w}=\frac{(s-p)^{w} y(s)}{(s+1)(s+b)^{w}},
$$

and $Y_{n w}(\infty)=Y_{n}(\infty)=1$. We re-calculate $\mid \Theta(s)\|=\| s\left[Y_{n w}(s) Y_{n}(\infty)^{-1}-I\right]+s Y_{d}(s) Y_{n}(\infty)^{-1} \| \leq$ $\left\|s\left[Y_{n w}(s) Y_{n}(\infty)^{-1}-I\right]\right\|+\left\|s Y_{d}(s) Y_{n}(\infty)^{-1}\right\|=:$ $\left\|\Theta_{n}(s)\right\|+\left\|\Theta_{d}(s)\right\|$. A condition on $\alpha$ more conservative than (9) is $\alpha>r\left\|\Theta_{n}(s)\right\|+r\left\|\Theta_{d}(s)\right\|$. For example, if $p=0.5$ and $w=1$, then $r=2$ and $\|\Theta(s)\|=14.5$ for $h_{1} \in[0.1,2.5]$. Therefore, (9) holds if we choose $\alpha=30$. The controller in (10) is then given by $C(s)=\frac{900(s+2)}{s(s+60)}$.

Example 2: For $h_{2}=\frac{\pi}{2} h_{1}$, consider

$$
\begin{aligned}
& \widehat{G}(s)=\widehat{Y}(s)^{-1} X(s) \\
& =\left[\begin{array}{cc}
\frac{s+1-2 e^{-h_{1} s}}{s+0.5} & \frac{s-1}{s+1} \\
0 & \frac{s-3 e^{-h_{2} s}}{s+2}
\end{array}\right]^{-1}\left[\begin{array}{cc}
\frac{1}{s+0.5} & 0 \\
\frac{1}{s^{2}+2 s+2} & \frac{1}{s+2}
\end{array}\right] .
\end{aligned}
$$

The MIMO plant $\widehat{G}$ is in the class in Section III-B. We have

$$
\begin{gathered}
\widehat{Y}(\infty)=Y_{n}(\infty)=\left[\begin{array}{cc}
1 & 1 \\
0 & 1
\end{array}\right], r_{1}=r_{2}=1 \text {. We compute } \\
\Theta(s)=s\left[\begin{array}{cc}
\frac{0.5-2 e^{-h_{1} s}}{s+0.5} & \frac{-2\left(1+e^{-h_{1} s}\right)}{s+1} \\
0 & \frac{-\left(2+3 e^{-h_{2} s}\right)}{s+1}
\end{array}\right] .
\end{gathered}
$$

It can be shown that for all $h_{1} \in[0.01,2.5],(18)$ is satisfied for $\alpha=5$. Hence $C(s)$ as in (20) stabilizes the given $\widehat{G}$ :

$$
C(s)=5\left[\begin{array}{cc}
\frac{s+0.5}{s} & \frac{s+0.5}{s} \\
\frac{-(s+0.5)(s+2)}{s\left(s^{2}+2 s+2\right)} & \frac{(s+2)\left(s^{2}+s+1.5\right)}{s\left(s^{2}+2 s+2\right)}
\end{array}\right]
$$

Example 3: Consider the following plant $\widehat{G}$, which is in the class considered in Section III-C:

$$
\widehat{G}(s)=\frac{K(s+z) e^{-h s}}{s^{\gamma_{1}}\left(s+p_{1}\right)\left(s+p_{2}\right)},
$$

where $\gamma_{1} \geq 1, p_{1}, p_{2} \in \mathbb{R}_{+}, z \in \mathbb{R} \backslash\{0\}$, and $h \geq 0$; Note that $z$ may be positive or negative, i.e., $\widehat{G}$ may have a finite zero in the right-half complex plane. Write $\widehat{G}=Y^{-1} \widehat{X}$ as

$$
\widehat{G}=Y^{-1} \widehat{X}=\left(\frac{s^{\gamma_{1}}}{(s+b)^{\gamma_{1}}}\right)^{-1}\left(\frac{K(s+z) e^{-h s}}{(s+b)^{\gamma_{1}}\left(s+p_{1}\right)\left(s+p_{2}\right)}\right)
$$

for any $b \in \mathbb{R}_{+}$. With $X(0)^{-1}=\frac{b^{\gamma_{1}} p_{1} p_{2}}{K z}$, and $\Phi$ as in (26), let $\beta \in \mathbb{R}_{+}$satisfy (27), i.e., $\beta<\frac{1}{\gamma_{1}}\|\Phi\|^{-1}$, where

$$
\|\Phi\|^{-1}=\left\|\frac{1}{s}\left[\frac{b^{\gamma_{1}} p_{1} p_{2} K(s+z) e^{-h s}}{K z(s+b)^{\gamma_{1}}\left(s+p_{1}\right)\left(s+p_{2}\right)}-1\right]\right\|^{-1} .
$$

Then $C=X(0)^{-1} \frac{\left[(s+\beta)^{\gamma_{1}}-s^{\gamma_{1}}\right]}{s^{\gamma_{1}}} Y=\frac{a p_{1} p_{2}\left[(s+\beta)^{\gamma_{1}}-s^{\gamma_{1}}\right]}{K z(s+b)^{\gamma_{1}}}$ as in (29) is a controller that stabilizes $\widehat{G}$. The controller $C$ is stable, and its order is $\gamma_{1}$, the same as the number of poles of $\widehat{G}$ at $s=0$, which is less than the plant's order. Let $\beta \in$ $\mathbb{R}_{+}$satisfy (30), i.e., $\beta<\frac{1}{1+\gamma_{1}}\|\Phi\|^{-1}$. Then an integralaction controller $\widetilde{C}$ as in (32) that stabilizes $\widehat{G}$ is $\widetilde{C}=$ $X(0)^{-1} \frac{\left[(s+\beta)^{\gamma_{1}+1}-s^{\gamma_{1}+1}\right]}{s^{\gamma_{1}+1}} Y=\frac{b^{\gamma_{1}} p_{1} p_{2}\left[(s+\beta)^{\gamma_{1}+1}-s^{\gamma_{1}+1}\right]}{K z s(s+b)^{\gamma_{1}}}$. For example, if $\gamma_{1}=1$, then $C$ and $\widetilde{C}$ become $C=$ $X(0)^{-1} \frac{\beta}{s} Y=\frac{b p_{1} p_{2} \beta}{K z(s+b)}$, where $\beta<\|\Phi\|^{-1}$, and $\widetilde{C}=$ $X(0)^{-1} \frac{\left(2 \beta s+\beta^{2}\right)}{s^{2}} Y=\frac{b p_{1} p_{2}\left(2 \beta s+\beta^{2}\right)}{K z s(s+b)}$, where $\beta<\frac{1}{2}\|\Phi\|^{-1}$.

\section{CONCLUSiOnS}

We proposed finite-dimensional controller designs for certain classes of SISO and MIMO systems subject to delays. These designs achieve closed-loop stability and integralaction. The controller order matches the relative degree of the finite-dimensional part of the plant for the plants in Sections III-A-III-B or the number of plant poles at the origin in Section III-C. Performance specifications beyond asymptotic tracking of constant references are not within the scope of this study. Future work will focus on expanding the plant classes to those that allow finite right-half plane zeros while not restricting the location of unstable poles.

\section{REFERENCES}

[1] C. Bonnet, J. R. Partington, "Analysis of fractional delay systems of retarded and neutral type," Automatica, vol. 38, pp. 11331138, 2002.

[2] C. Bonnet, J.R. Partington, "Stabilization of some fractional delay systems of neutral type," Automatica, vol. 43, pp. 2047-2053, 2007.

[3] R.F. Curtain, K. Glover, "Robust stabilization of infinite-dimensional systems by finite-dimensional controllers," Systems Control Letters, 7 (1), pp. 4147, 1986.

[4] R.F. Curtain, H. Zwart, An Introduction to Infinite-dimensional Linear Systems Theory, Texts in Applied Mathematics, Vol. 21, Springer, New York, 1995.

[5] C. Foias, H. Özbay, A. Tannenbaum, Robust Control of Infinite Dimensional Systems, LNCIS 209, Springer-Verlag, London, 1996.

[6] B. A. Francis and W. A. Wonham, "The internal model principle for linear multivariable regulators," Applied Mathematics \& Optimization, 2:2, pp. 170-195, 1975.

[7] K. Gu, V. L. Kharitonov, J. Chen, Stability of Time-Delay Systems, Birkhäuser, Boston, 2003.

[8] A. N. Gündeş, H. Özbay, and A. B. Özgüler, "PID controller synthesis for a class of unstable MIMO plants with I/O Delays," Automatica, vol. 43, no. 1, pp. 135-142, 2007.

[9] A. N. Gündeş, H. Özbay, "Reliable decentralized control of delayed MIMO plants," Int. Jour. Control, vol. 83, no. 3, pp. 516-526, 2010.

[10] S. I. Niculescu, Delay Effects on Stability: A Robust Control Approach, LNCIS, vol. 269, Heidelberg: Springer-Verlag, 2001.

[11] H. Özbay, A. N. Gündeș, "Integral action controllers for systems with time delays," in Topics in Time Delay Systems. Analysis, Algorithms and Control, LNCIS 388, J. J. Loiseau, W. Michiels, S-I. Niculescu, R. Sipahi (Eds.), pp. 197-208, Springer-Verlag, London, 2009.

[12] S. Skogestad, "Simple analytic rules for model reduction and PID controller tuning," Jour. Process Control, vol. 13, pp. 291-309, 2003.

[13] G. J. Silva, A. Datta, S. P. Bhattacharyya, PID Controllers for TimeDelay Systems, Birkhäuser, Boston, 2005. 